\title{
Berberine alleviates dextran sodium sulfate-induced colitis by improving intestinal barrier function and reducing inflammation and oxidative stress
}

\author{
LI-CHAO ZHANG ${ }^{1 *}$, YUE WANG ${ }^{2 *}$, LING-CHANG TONG ${ }^{3 *}$, SHENG SUN $^{3}$, WEI-YE LIU ${ }^{3}$, \\ SU ZHANG ${ }^{1,3}$, RONG-MEI WANG ${ }^{3}$, ZHI-BIN WANG ${ }^{3}$ and LING LI $^{3}$ \\ ${ }^{1}$ Department of Pharmacy, Shanghai Municipal Hospital of Traditional Chinese Medicine, Shanghai 200071; \\ ${ }^{2}$ Department of Pharmacy, Ningxia Medical University, Yinchuan, Ningxia 750004; ${ }^{3}$ Department of Pharmacology, \\ College of Pharmacy, The Second Military Medical University, Shanghai 200433, P.R. China
}

Received December 27, 2015; Accepted January 6, 2017

DOI: 10.3892/etm.2017.4402

\begin{abstract}
Berberine has demonstrated efficacy in alleviating experimental colitis in vivo and in vitro. However, the anti-colitis mechanisms of berberine that enable it to promote intestinal barrier function in vivo remain unclear. The present study aimed to evaluate the effect of berberine on intestinal epithelial barrier function, expression of tight junction proteins and the levels of inflammatory and oxidative stress factors in the intestinal mucosa of dextran sulfate sodium (DSS)-induced colitis mice. Berberine $(100 \mathrm{mg} / \mathrm{kg})$ was administered for five days to mice with established colitis, induced by administration of DSS (3\% w/v) for six days. Intestinal barrier function and the presence of proinflammatory factors, oxidative stress and active signaling pathways in the colon were determined principally by western blotting and reverse transcription-quantitative polymerase chain reaction. It was observed that berberine reduced weight loss, shortening of the colon and colon damage in DSS-colitis mice. In addition, berberine significantly inhibited the increase of fluorescein isothiocyanate-dextran in serum and the decrease of zonula occluden-1 (also known as tight junction protein-1), occludin and epithelial cadherin expression in colonic tissue, relative to a DSS-treated control group.
\end{abstract}

Correspondence to: $\mathrm{Dr}$ Li-Chao Zhang, Department of Pharmacy, Shanghai Municipal Hospital of Traditional Chinese Medicine, 274 Zhi Jiang Middle Road, Shanghai 200071, P.R. China E-mail: changhaiskin@163.com

Dr Ling Li, Department of Pharmacology, College of Pharmacy, The Second Military Medical University, 325 Guohe Road, Shanghai 200433, P.R. China

E-mail: lingli_z163@163.com

*Contributed equally

Key words: ulcerative colitis, berberine, intestinal barrier function, tight junction protein, dextran sulfate sodium, inflammation
Berberine also significantly inhibited the expression of interleukin (IL)-1 $\beta$, IL- 6 and tumor necrosis factor- $\alpha$ mRNA and phosphorylation of signal transducer and activator of transcription 3. Furthermore, berberine reduced the levels of myeloperoxidase and increased the levels of superoxide dismutase and catalase in colon and serum samples relative to the control group. The expression of cluster of differentiation 68 in the colon of colitis mice was also reduced by berberine. Collectively, these data suggest that berberine alleviates colitis principally by improving intestinal barrier function and promoting anti-inflammatory and antioxidative stress responses. In turn these effects inhibit macrophage infiltration into the colon and thus may be central to the anti-colitis activity of berberine.

\section{Introduction}

Inflammatory bowel disease (IBD), mainly comprised of ulcerative colitis (UC) and Crohn's disease (CD), is characterized by chronic relapsing inflammation associated with architectural distortion of the intestinal epithelium and impaired mucosal barrier function (1). The pathogenesis of IBD is linked to a complex interaction between a number of risk factors, including susceptibility genes, environmental factors, the immune system and microorganisms (2). IBD is prevalent in the western world, and in European countries, the incidence rates for CD and UC are 0.5-10.6 and 0.9-24.3/ 10,000 individuals, respectively (3). Traditional drugs such as glucocorticoids, sulfasalazine and immunosuppressants are used for the treatment and maintenance of UC, and drugs such as aminosalicylates, antibiotics, corticosteroids and immunosuppressants are used for the treatment of $\mathrm{CD}$, though these are limited by their incomplete clinical efficacy and side effects (4,5).

Berberine is an isoquinoline alkaloid and one of the main components of the herb Coptidis rhizoma (species: Coptis chinensis Franch, genus: Var. asperma Don, family: Ranunculaceae). In traditional Chinese medicine, berberine is considered to be useful in the treatment of patients with gastroenteritis, abdominal pain and/or diarrhea, due to its low cost and small number of side effects (6). Previous studies have 
indicated a number of pharmacologic effects of berberine, including antimicrobial, antiprotozoal, antidiarrhea and anticholera toxin, cholesterol-lowering and anti-inflammatory activities $(7,8)$. In addition, it has been observed in vivo that berberine alleviated experimental colitis induced by dextran sulfate sodium (DSS) or trinitrobenzene sulfonic acid (TNBS) in mice or rats, attributed to the anti-inflammatory and anti-oxidative properties of the compound (9-11). Furthermore, results of previous studies have indicated that berberine may promote barrier function in human retinal pigment epithelial cells, endothelial cells and Caco-2 human epithelial colorectal adenocarcinoma cells (12-15). However, the in vivo anti-colitis activity of berberine regarding intestinal barrier function is not well studied.

Therefore, the present study aimed to evaluate the anti-colitis effects of berberine on intestinal barrier function and the expression of tight junction proteins [zonula occluden-1 (ZO-1), occludin and epithelial (E)-cadherin] in DSS-induced colitis mice. Furthermore, the effect of berberine on inflammation and oxidative stress, and its underlying mechanisms of action, were investigated.

\section{Materials and methods}

Materials. DSS (36-50 kDa) was purchased from MP Biomedicals, LLC (Santa Ana, CA, USA). Berberine was purchased from Sigma-Aldrich (Merck KGaA, Darmstadt, Germany) and TRIzol reagent was purchased from Invitrogen (Thermo Fisher Scientific, Inc., Waltham, MA, USA). FastStart Universal SYBR-Green Master (Rox) and PrimeScript Reverse Transcription (RT) Master Mix Perfect Real-Time kits were purchased from Roche Diagnostics GmbH (Mannheim, Germany) and Takara Biotechnology Co., Ltd. (Dalian, China) respectively. Superoxide dismutase (SOD), catalase (CAT) and myeloperoxidase (MPO) activity assay kits were purchased from Nanjing Jiancheng Bioengineering Institute (Nanjing, China).

Primary antibodies for occludin and E-cadherin were from Thermo Fisher Scientific, Inc. and Cell Signaling Technology, Inc. (Danvers, MA, USA), respectively. Primary antibodies for ZO-1 and $\beta$-actin were from Santa Cruz Biotechnology, Inc. (Dallas, TX, USA) and Sigma-Aldrich (Merck KGaA), respectively. Mouse monoclonal anti-signal transducer and activator of transcription 3 (STAT3; 124H6) and anti-phosphorylated (p)-STAT-3 (p-Tyr705) antibodies were from Cell Signaling Technology, Inc. Mouse monoclonal anti-cluster of differentiation (CD68) antibody was from Abcam (Cambridge, England).

Animals. A total of $40 \mathrm{C} 57 \mathrm{BL} / 6 \mathrm{~J}$ male mice (six weeks old) weighing 18-22 g were purchased from Shanghai SLAC Laboratory Animal Co., Ltd. (Shanghai, China). Mice were kept under an automated 12-h light-dark cycle at a controlled temperature of $22 \pm 2^{\circ} \mathrm{C}$, relative humidity of $50-60 \%$ and had ad libitum access to a standard dry diet and tap water. The animals received humane care and experimental procedures were performed in accordance with the health and care of experimental animals guidelines of the Second Military Medical University (Shanghai, China).

DSS-induced colitis. Mice were divided into the following three groups: i) Control group (group 1); ii) DSS-induced colitis group (group 2); and iii) DSS-induced colitis plus berberine-treated group (group 3). Each group consisted of ten mice. Mice in group 1 received drinking water alone and were used as negative controls. Colitis was induced in mice of groups 2 and 3 by administration of DSS (3\% w/v, dissolved in drinking water) for six days. Berberine $(100 \mathrm{mg} / \mathrm{kg}$, dissolved in distilled water) was administered to group 3 mice via oral gavage once a day for a period of five days after the six days DSS treatment period. Daily assessments of weight loss, stool consistency and fecal blood, as indicators of disease activity index, were measured. The mice in all groups were sacrificed 11 days after initiation of experimental treatments, following the final administration of berberine to group 3 mice. Prior to sacrifice by cervical dislocation, mice were anesthetized with an intraperitoneal injection of $4 \%(\mathrm{w} / \mathrm{v})$ chloral hydrate (Sinopharm Chemical Reagent Co., Ltd., Shanghai, China) in order to collect blood samples. Colonic tissues were then removed for histopathological examination.

Histopathological examination. Colon sections were taken from the mice for histological examination. The length of the colon from the anus to appendix was measured by a ruler, then segments of colon were excised, fixed in $10 \%$ phosphate-buffered saline (PBS)-buffered formalin at room temperature for $24 \mathrm{~h}$, then embedded in paraffin. Sections measuring 5-10 $\mu \mathrm{m}$ in thickness were cut, placed on glass slides and stained with hematoxylin and eosin (H\&E). Histopathological changes were observed under a light microscope and graded semiquantitatively using a histological injury scale, as described by Wirtz et al (16). The scores for histological changes were as follows: 0, no evidence of inflammation; 1, low level inflammation with scattered mononuclear cells (1-2 foci); 2 , moderate inflammation with multiple foci of mononuclear cells; 3 , high level inflammation with increased vascular density and marked wall thickening; and 4, maximal inflammation with transmural leukocyte infiltration and loss of goblet cells.

Measurement of MPO level in colon and serum samples. The ability of MPO to modulate levels of hydrogen peroxide is considered to be an indicator of MPO activity (17). MPO activity was determined using an MPO activity assay kit, according to a modified method of the manufacturer's protocol, as follows: Freshly excised colon was rinsed with PBS, homogenized in tissue lysis buffer and centrifuged at $12,000 \mathrm{x} \mathrm{g}$ and $4^{\circ} \mathrm{C}$ for $15 \mathrm{~min}$ to isolate proteins. Serum was isolated without lysis buffer for later analysis of serum MPO. Blood samples were then centrifuged at $3,500 \times \mathrm{g}$ for $20 \mathrm{~min}$ at $4^{\circ} \mathrm{C}$. Pellets were resuspended in PBS containing $0.5 \%$ hexadecyl-trimethylammonium bromide (Nanjing Jiancheng Bioengineering Institute, Nanjing, China), then freeze-thawed three times. MPO activity was determined by measuring the absorbance of the resulting solutions at $460 \mathrm{~nm}$ with an automatic microplate reader at $37^{\circ} \mathrm{C}$. Protein concentration was then measured quantitatively using a Bicinchoninic Acid-100 Protein Determination kit (Shenneng Bocai Biotechnology Co., Ltd., Shanghai, China), according to the manufacturer's protocol. As the degradation of peroxide by MPO results in a color change, MPO activity was defined as the quantity of enzyme degrading $1 \mu \mathrm{mol} / \mathrm{ml}$ peroxide at $37^{\circ} \mathrm{C}$, as determined from absorbance measurements, expressed as $\mathrm{U} /$ $\mathrm{mg}$ of colon protein. 
Detection of SOD and CAT levels in colon and serum samples. Freshly excised colon was rinsed with PBS, homogenized in tissue lysis buffer and centrifuged at $12,000 \times \mathrm{x}$ and $4^{\circ} \mathrm{C}$ for 15 min. Individual activities of SOD and CAT in tissue lysates and serum were then measured using SOD and CAT activity assay kits, respectively, according to the manufacturer's protocol. Individual activities of SOD and CAT were measured at 450 and $405 \mathrm{~nm}$, respectively, with a microplate fluorometer. Total protein concentration was measured using the Bradford method (Beyotime Institute of Biotechnology, Haimen, China). The concentration of SOD and CAT in colon and serum samples was presented as $\mathrm{pg} / \mathrm{mg}$ of total protein.

Intestinal permeability. Intestinal epithelial permeability in vivo was determined according to a previously described method (18). Briefly, mice were fasted overnight and fluorescein isothiocyanate (FITC)-dextran solution (4 kDa, $600 \mathrm{mg} / \mathrm{kg}$ ) was delivered via gavage the following day. Mice were then anesthetized with $4 \%$ (w/v) chloral hydrate (intraperitoneal injection) $4 \mathrm{~h}$ after FITC-dextran administration. Abdominal cavities were exposed with surgical instruments and blood was harvested via cardiac puncture with a 1-ml injector. Blood samples were then centrifuged at $3,500 \times \mathrm{g}$ and $4^{\circ} \mathrm{C}$ for $20 \mathrm{~min}$. Serum levels of FITC were measured at 480 and $520 \mathrm{~nm}$ using a microplate fluorometer.

Protein extraction and western blot analysis. Immunoblotting to detect colonic proteins was performed according to a previously described method (19). Briefly, colonic tissues were removed and washed in PBS. Whole tissue was then cut into pieces and homogenized in five volumes of ice-cold radioimmunoprecipitation assay buffer (Kangcheng Pharmaceutical Co., Ltd., Guangzhou, China) containing $1 \mu 1$ protease and phosphatase inhibitors. The mixture was then centrifuged at $14,000 \times \mathrm{g}$ and $4^{\circ} \mathrm{C}$ for $15 \mathrm{~min}$, and protein concentration of the resulting supernatants was determined by a Bradford assay kit (Beyotime Institute of Biotechnology), according to the manufacturer's protocol. Equal quantities of protein were separated by a $10 \%(\mathrm{w} / \mathrm{v})$ SDS-PAGE gel ( $20 \mu \mathrm{g} / \mathrm{lane})$ and transferred to nitrocellulose membranes. The membranes were blocked for $3 \mathrm{~h}$ at room temperature with 5\% blocking reagent, then incubated overnight at $4^{\circ} \mathrm{C}$ with anti-STAT3 antibody $(1: 1,000)$, anti-p-STAT3 antibody $(1: 1,000)$, anti-occludin antibody $(1: 2,000)$, anti-E-cadherin antibody $(1: 2,000)$, anti-ZO-1 antibody $(1: 1,000)$ and $\beta$-actin antibody $(1: 10,000)$, according to the manufacturer's instructions. After washing with PBS-Tween 20, membranes were incubated with Cyanine ${ }^{\mathrm{TM}}$-conjugated affinipure donkey anti-rabbit immunoglobulin (Ig)-G [heavy (H) and light (L) chains; cat. no. 712-167-003; Jackson ImmunoResearch Laboratories, Inc., West Grove, PA, USA] and HiLyte $488^{\mathrm{TM}}$-conjugated affinipure donkey anti-goat IgG (H+L chains; cat. no. 705-167-003; Jackson Immunoresearch Laboratories, Inc.) at room temperature for $1 \mathrm{~h}$. Protein bands were visualized and quantified with an Odyssey Infrared Imaging 3.0 system (LI-COR Biosciences, Lincoln, NE, USA). $\beta$-actin was used as a protein loading control. Immunoblotting experiments were repeated at least three times.

$R N A$ isolation and RT-quantitative polymerase chain reaction $(q P C R)$. Total RNA was extracted from tissue homogenates of mouse colon with TRIzol reagent, according to the manufacturer's protocol. RNA was reverse transcribed into cDNA with a PrimeScript Reverse Transcription (RT) Master Mix Perfect Real-Time kit, according to the manufacturer's protocol. Resulting cDNA was then used as a template for qPCR. qPCR was performed using an ABI 7500 real-time PCR system (Applied Biosystems; Thermo Fisher Scientific, Inc., Foster City, CA, USA). The primer sequences were as follows: GAPDH, forward, 5'-GTATGACTCCACTCACGGCAAA-3' and reverse, 5'-GGTCTCGCTCCTGGAAGATG-3'; interleukin (IL)-1 $\beta$, forward, 5'-CTCACA AGCAGAGCAC AAGC-3' and reverse, 5'-CAGTCCAGCCCATACTTT AGG-3'; IL-6, forward, 5'-CGGAGAGGAGACTTCACA GAG-3' and reverse, 5'-CATTTCCACGATTTCCCAGA-3'; tumor necrosis factor (TNF)- $\alpha$, forward, CATTTCCACGATT TCCCAGA-3' and reverse, 5'-GGAAAGCCCATTTGAGT CCT-3'. GAPDH was used as an internal control and RNA products were quantified by the DDCq method (20). RT-qPCR experiments were performed at least three times.

Immunofluorescence assay of macrophage infiltration. An immunofluorescence assay to detect macrophage infiltration in mouse colonic mucosa was performed according to a previously described method (21). Briefly, after colons were excised and rinsed with PBS, colon tissues were fixed in 10\% PBS-buffered formalin at room temperature for $24 \mathrm{~h}$, then embedded in paraffin, cut into $5 \mu \mathrm{m}$ sections and mounted on glass slides. Sections were subsequently deparaffinized in xylene and rehydrated in a graded series of ethanol solutions (50 75, 85, 95 and 100\%, $3 \mathrm{~min} /$ solution). Sections were then blocked with $5 \%$ bovine serum albumin (BSA; Beyotime Institute of Biotechnology) in Tris-buffered saline (TBS) for $90 \mathrm{~min}$ at room temperature, then immunostained overnight at $4^{\circ} \mathrm{C}$ with anti-CD68 antibody (1:100) and 5\% BSA in TBS. Following immunostaining, sections were washed three times with TBS, then incubated with Alexa Fluor 488-conjugated secondary antibody (1:200; cat. no. 715-547-003; Jackson Immunoresearch Laboratories, USA) in TBS for $2 \mathrm{~h}$ at room temperature in the dark. Sections were then mounted with mounting medium containing $5 \mathrm{mg} / \mathrm{ml}$ DAPI (Beyotime Institute of Biotechnology, Shanghai, China) for nuclear counterstaining and visualized under an Olympus IX71 inverted fluorescent microscope (Olympus Corporation, Tokyo, Japan).

Statistical analysis. Quantified data are presented as the mean \pm standard deviation. Differences between two groups were determined with the Student's $t$-test. Statistical analysis was performed using SPSS 11.0 software (SPSS, Inc., Chicago, IL, USA) and $\mathrm{P}<0.05$ was considered to indicate a statistically significant difference.

\section{Results}

Berberine reduces weight loss, shortening of the colon and colon damage in DSS-colitis mice. Relative to the untreated control group (group 1), DSS-induced colitis mice (groups 2 and 3) exhibited marked weight loss five to seven days after initiation of a six days DSS treatment (Fig. 1A). After day 8, group 2 and 3 mice exhibited recovery of body weight, however body weight recovery was significantly greater on days 8,9 
A

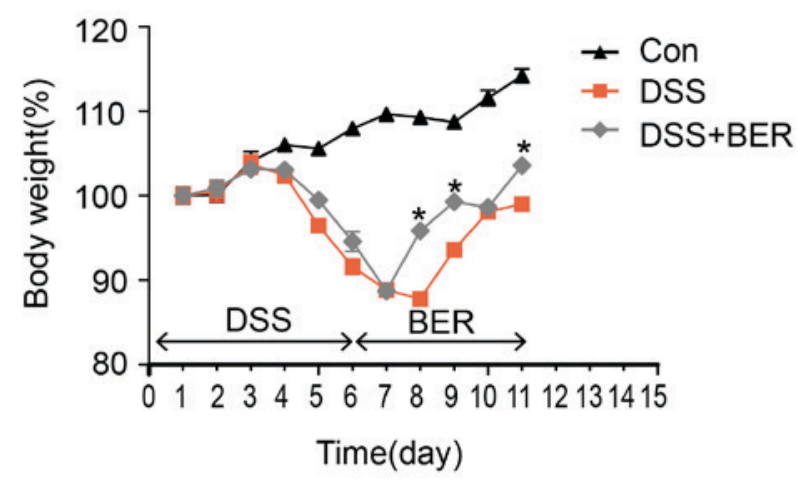

C

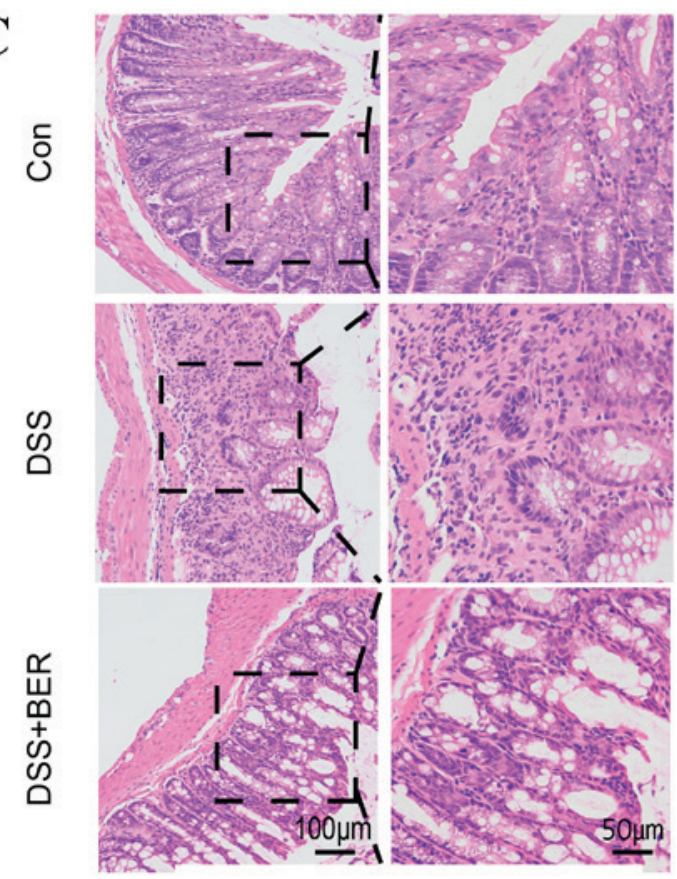

B
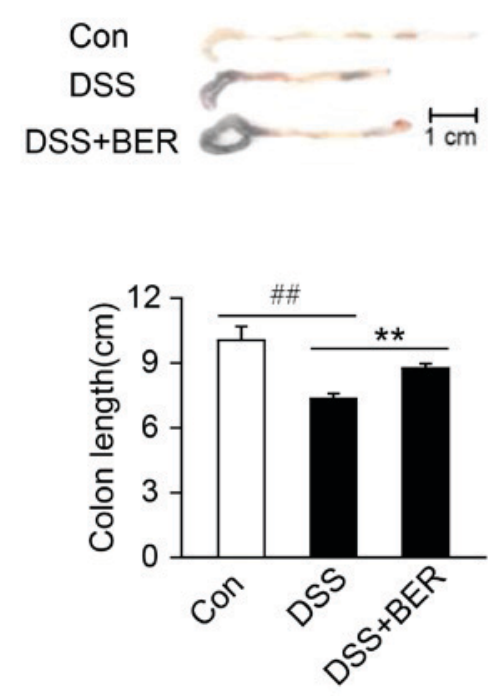

D

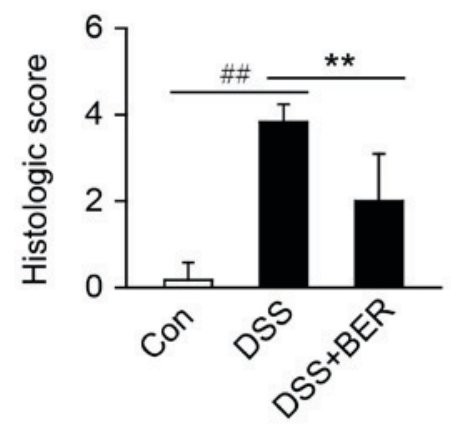

Figure 1. Effects of berberine on body weight, colon length and histological evaluation in DSS-induced colitis mice. Mice were administered with a $3 \%$ DSS solution for six days (days 1 to 6$)$. BER $(100 \mathrm{mg} / \mathrm{kg}$ ) was then administered for five days (days 7 to 11$)$ to the DSS+BER group. Control mice received water alone. On day 12, (A) body weight and (B) colon length were measured. (C) Colon damage was subsequently assessed by hematoxylin and eosin staining of paraffin-embedded colon sections. Images shown are representative of 10 mice in each group. Right column images are magnifications of the highlighted regions in left column images. (D) Colon injury scores were allocated following histological examination. Data are presented as the mean \pm standard deviation. ${ }^{*} \mathrm{P}<0.05$ and ${ }^{* *} \mathrm{P}<0.01$ vs. DSS group, ${ }^{\# \#} \mathrm{P}<0.01$ vs. control group; $\mathrm{n}=10$. DSS, dextran sulfate sodium; BER, berberine; Con, control.

and 11 for group 3 mice administered with berberine (day 7), relative to berberine-untreated group 2 mice $(\mathrm{P}<0.05$; Fig. $1 \mathrm{~A})$.

Colon length was also measured as an indicator of colitis. Relative to that of the control group, colon length was significantly decreased in group $2 \mathrm{DSS}$-colitis mice $(\mathrm{P}<0.01)$. In turn, relative to that of group 2 mice, colon length was significantly increased in group $3 \mathrm{DSS}$-colitis mice administered with berberine $(\mathrm{P}<0.01$; Fig. $1 \mathrm{~B})$.

Histological and morphological characteristics of the colons were subsequently examined by H\&E staining. In the control group, colon tissue exhibited normal crypt morphology and goblet cell count, and absence of mucosal thickening and ulcerations (Fig. 1C). By contrast, colons of group 2 DSS-colitis mice exhibited superficial ulcerations, loss of goblet cells, neutrophil infiltration and goblet cell damage. Thus, group 2 colon tissue received a significantly higher histological damage score than that of the control group ( $\mathrm{P}<0.01$; Fig. $1 \mathrm{C})$. In turn, relative to the colon tissue of group 2 mice, colons of berberine-treated group 3 colitis mice displayed reduced neutrophil infiltration, minimal goblet cell loss and thus a significantly lower histological damage score $(\mathrm{P}<0.01$; Fig. 1C).

Berberine improves intestinal barrier function in DSSinduced colitis mice. Intestinal epithelial tight junctions serve a key role in protecting against inflammation, and disrupted tight junctions are a main cause of intestinal barrier dysfunction and inflammation (22). Therefore, the present study evaluated the effects of berberine on intestinal permeability in vivo using FITC-dextran. It was observed that the level of FITC-dextran in the serum of group 2 DSS-colitis mice was significantly higher than that in control mice $(\mathrm{P}<0.01$; Fig. $2 \mathrm{~A})$. In turn, the 
A

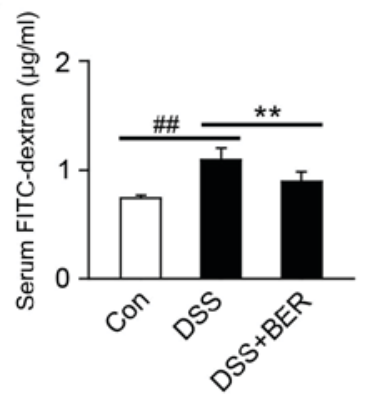

C

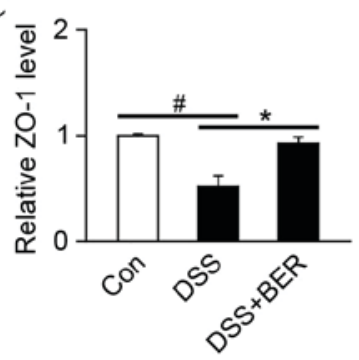

B

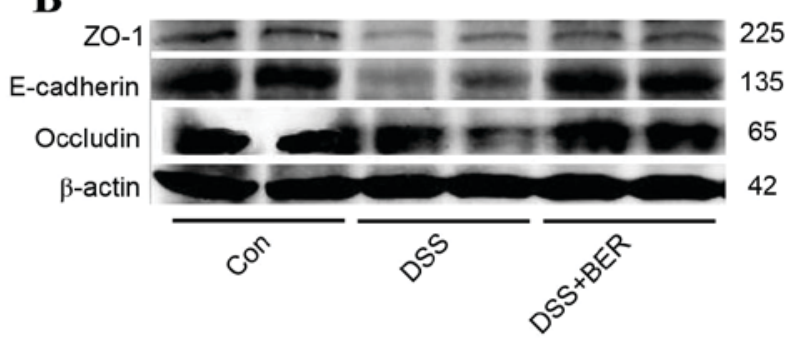

D

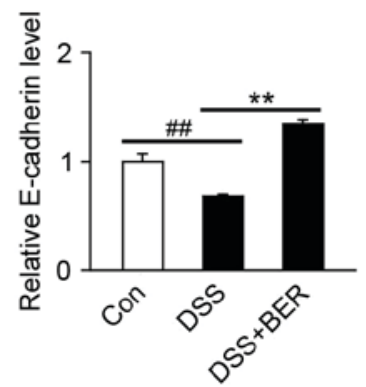

E

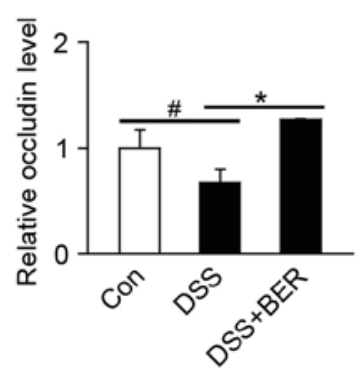

Figure 2. Effects of berberine on intestinal barrier function in DSS-colitis mice. (A) Levels of FITC-dextran in the serum of DSS-colitis mice administered with FITC-dextran were measured as an indicator of intestinal permeability. $n=10$. (B) Homogenates of colonic tissue were analyzed by western blotting to detect the expression of tight junction-associated proteins (ZO-1, E-cadherin and occludin). Representative blots from 4 samples of three independent experiments are shown and $\beta$-actin was used as a loading control. Levels of (C) ZO-1, (D) E-cadherin and (E) occludin expression were subsequently quantified. Data are presented as the mean \pm standard deviation. ${ }^{*} \mathrm{P}<0.05$ and ${ }^{* *} \mathrm{P}<0.01$ vs. DSS group, ${ }^{\#} \mathrm{P}<0.05$ and ${ }^{\# \#} \mathrm{P}<0.01$ vs. control group; $\mathrm{n}=10 \mathrm{DSS}$, dextran sulfate sodium; FITC, fluorescein isothiocyanate; ZO-1, zonula occluden-1; E-cadherin, epithelial cadherin; BER, berberine; Con, control.

elevated serum levels of FITC-dextran in DSS-colitis mice were significantly reduced by berberine treatment in group 3 mice $(\mathrm{P}<0.01$; Fig. $2 \mathrm{~A})$.

To verify the involvement of epithelial tight junctions, the levels of tight junction-associated protein expression, namely ZO-1, E-cadherin and occludin, were determined by western blotting (Fig. 2B). Relative to control mice, significant decreases in the levels of colonic ZO-1, E-cadherin and occludin and were identified in group 2 DSS-colitis mice $(\mathrm{P}<0.05, \mathrm{P}<0.01$ and $\mathrm{P}<0.05$, respectively; Fig. 2C-E). In turn, the reduced levels of $\mathrm{ZO}-1$, E-cadherin and occluding expression in DSS-colitis mice were significantly increased by berberine treatment in group 3 mice $(\mathrm{P}<0.05, \mathrm{P}<0.01$ and $\mathrm{P}<0.05$, respectively; Fig. 2C-E).

Berberine reduces expression of proinflammatory cytokine $m R N A$ in DSS-colitis mice. Levels of the proinflammatory cytokines, IL-1 $\beta$, IL- 6 and TNF- $\alpha$, in the colon of DSS-colitis mice were measured at the mRNA level by RT-qPCR. It was observed that relative to control mice, levels of IL-1 $\beta$, IL- 6 and TNF- $\alpha$ mRNA in group 2 DSS-colitis mice were significantly increased $(\mathrm{P}<0.05, \mathrm{P}<0.05$ and $\mathrm{P}<0.01$, respectively; Fig. 2A-C). In turn, the elevated levels of IL- $1 \beta$, IL- 6 and TNF- $\alpha$ mRNA in DSS-colitis mice were significantly decreased by berberine treatment in group 3 mice (all $\mathrm{P}<0.05$; Fig. $3 \mathrm{~A}-\mathrm{C}$ ).

STAT3 is involved in colonic inflammation and is activated by a number of cytokines and growth factors, including as IL-6 and IL-17 $(23,24)$. Therefore, the effect of berberine on the phosphorylation of STAT3 in DSS-colitis mice was evaluated by western blot analysis (Fig. 3D). It was demonstrated that the levels of p-STAT3 in colonic tissue of DSS-colitis mice were significantly increased relative to control mice $(\mathrm{P}<0.01$;
Fig. 3D). In turn, the elevated levels of p-STAT3 in DSS-colitis mice were significantly decreased by berberine treatment in group 3 mice $(\mathrm{P}<0.05$; Fig. 3D).

Berberine regulates the oxidative stress response in DSS-colitis mice. MPO, SOD and CAT serve key roles in inflammation and oxidative stress (25). Therefore, their levels in the serum and colonic tissue of DSS-colitis mice were determined by enzyme activity assays (Fig. 4). Relative to control mice, it was observed that the levels of MPO in serum and colonic tissue of DSS-colitis mice were significantly higher (both $\mathrm{P}<0.01$; Fig. 4A and D). In turn, the elevated serum and colon levels of MPO in DSS-colitis mice were significantly decreased by berberine treatment in group 3 mice.

By contrast, significant decreases in the serum and colon levels of SOD (both $\mathrm{P}<0.01$; Fig. $4 \mathrm{~B}$ and E) and CAT $(\mathrm{P}<0.05$ and $\mathrm{P}<0.01$, respectively; Fig. $4 \mathrm{C}$ and F) were identified in group $2 \mathrm{DSS}$-colitis mice, relative to control mice. In turn, significant increases in the serum and colon levels of SOD (both $\mathrm{P}<0.05$; Fig. 4B and $\mathrm{E})$ and CAT $(\mathrm{P}<0.05$ and $\mathrm{P}<0.01$, respectively; Fig. $4 \mathrm{C}$ and $\mathrm{F}$ ) were observed following berberine treatment in group 3 colitis mice, relative to group 2 mice.

Berberine inhibits the expression of CD68 in DSS-colitis mice. CD68 may be used as a marker of macrophages (and monocytes) in the colon of mice. Therefore, the present study measured CD68 expression in colonic tissue of DSS-colitis mice in order to determine the level of macrophage infiltration. It was observed that CD68 expression was markedly increased in DSS-induced mice relative to control mice, and that subsequent berberine treatment reduced the elevated expression of CD68 in DSS-colitis mice (Fig. 5). 
A

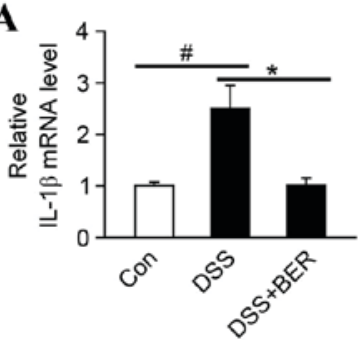

D

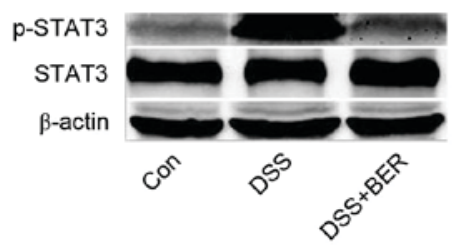

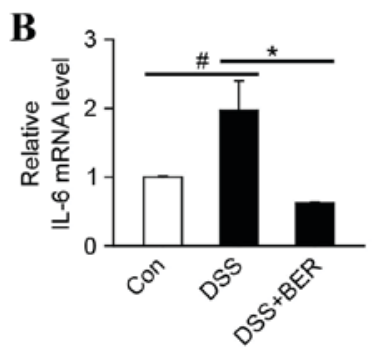
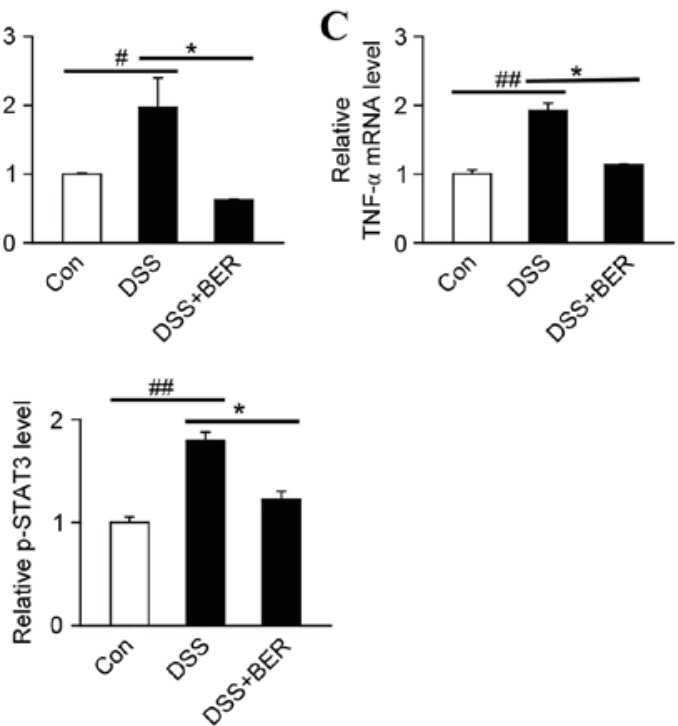

Figure 3. Effects of berberine on proinflammatory factor mRNA expression and STAT3 phosphorylation in the colon of DSS-colitis mice. Colonic tissue was analyzed by reverse transcription-quantitative polymerase chain reaction to measure the levels of (A) IL-1 $\beta$, (B) IL- 6 and (C) TNF- $\alpha$ mRA. Levels of proinflammatory factor expression in DSS and DSS+BER mice were measured relative to untreated control mice. (D) Levels of p-STAT3 were determined by western blot analysis. Representative blots of three independent experiments are shown and $\beta$-actin was used as a loading control. *P<0.05 vs. DSS group, ${ }^{\#} \mathrm{P}<0.05$ and ${ }^{\# \#} \mathrm{P}<0.01$ vs. control group; $\mathrm{n}=4$. STAT3, signal transducer and activator of transcription 3; DSS, dextran sulfate sodium; IL, interleukin; TNF- $\alpha$, tumor necrosis factor- $\alpha$ BER, berberine; Con, control; p-, phosphorylated.

A

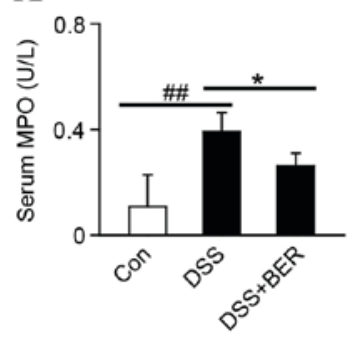

D

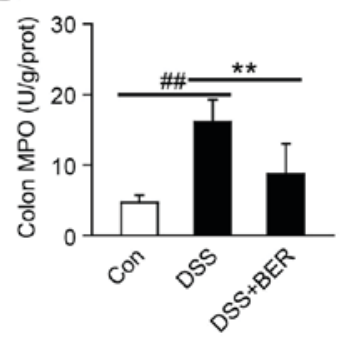

B

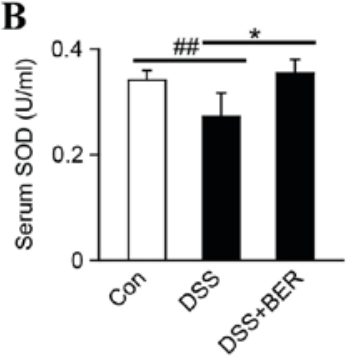

$\mathbf{E}$

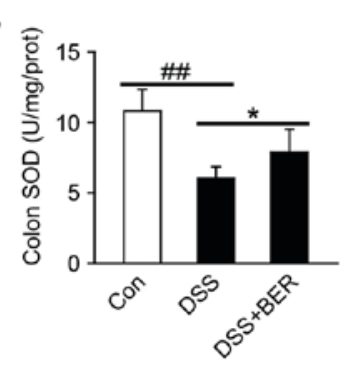

C

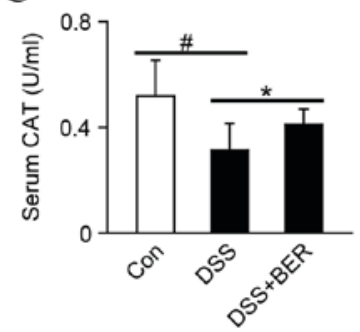

$\mathbf{F}$

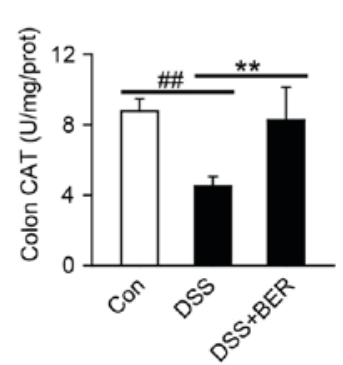

Figure 4. Effect of berberine on the oxidative stress response in DSS-colitis mice. As indicators of oxidative stress, levels of (A) MPO, (B) SOD and (C) CAT in the serum of DSS-colitis mice were measured by enzyme activity assays. Colonic tissues were also analyzed to determine the levels of (D) MPO, (E) SOD and (F) CAT. Data are presented as the mean \pm standard deviation. ${ }^{*} \mathrm{P}<0.05$ and ${ }^{* *} \mathrm{P}<0.01$ vs. DSS group, ${ }^{\#} \mathrm{P}<0.05$ and ${ }^{\# \#} \mathrm{P}<0.01$ vs. control group; $\mathrm{n}=10$. DSS, dextran sulfate sodium; MPO, myeloperoxidase; SOD, superoxide dismutase; CAT, catalase; BER, berberine; Con, control.

\section{Discussion}

The present study demonstrated that berberine treatment in DSS-colitis mice improved intestinal barrier function and inhibited the downregulation of tight junction proteins ZO-1, E-cadherin and occludin. Berberine treatment also decreased MPO activity and the expression of proinflammatory cytokine (IL-6, IL-1 $\beta$ and TNF- $\alpha$ ) mRNA in the colon. Furthermore, berberine stimulated the activity of CAT and SOD in the colon and serum, decreased the phosphorylation of colonic STAT3 and inhibited macrophage infiltration into the intestinal mucosa of DSS-colitis mice. Collectively, these results suggest that berberine alleviates DSS-induced colitis though regulation of intestinal permeability, inflammation and oxidative stress.

The intestinal epithelial barrier serves a key role in preventing luminal pathogens and antigenic molecules from penetrating the intestinal mucosa and contacting 

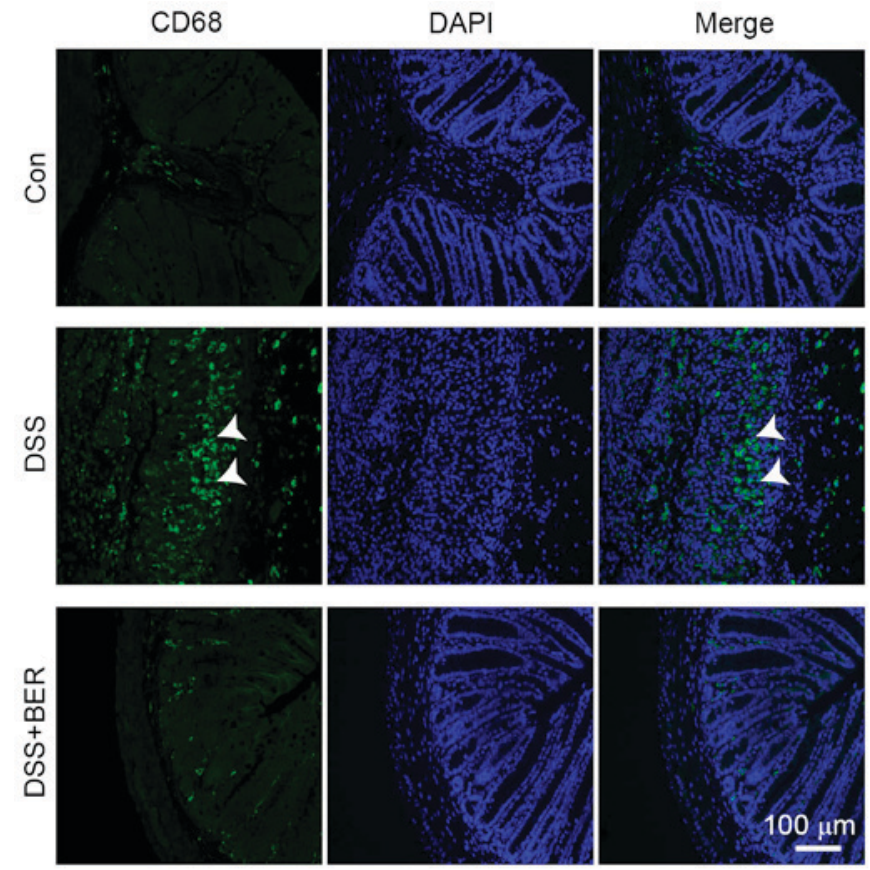

Figure 5. Effects of berberine on CD68-positive macrophages in the colon of DSS-colitis mice. Paraffin-embedded colon tissues were analyzed by immunofluorescence to detect macrophage infiltration in DSS-colitis mice. Expression of CD68 was used as a marker of macrophages and monocytes (green staining; white arrows). Cell nuclei were stained with DAPI (blue staining). Images are representative of 10 mice in each group. CD68, Cluster of Differentiation 68; DSS, dextran sulfate sodium; BER, berberine; Con, control.

the submucosal immune system (26). Defective intestinal epithelial barrier function is characterized by increased intestinal permeability (27). Normally, selective paracellular permeability exists across the intestinal epithelial monolayer, which is modulated by protein-based intercellular junctions, namely tight and adherent junctions (28). In the present study, occludin and E-cadherin were selected as representative proteins of tight junctions and adherence junctions, while ZO-1 was selected as a representative junction scaffolding protein (29). It was demonstrated that the levels of occludin, E-cadherin and ZO-1 expression were decreased, while the level of FITC-dextran in serum was increased, in DSS-colitis mice. These results are consistent with the downregulation of occludin, E-cadherin and ZO-1 observed in UC and CD patients (30-32). Furthermore, in vivo and in vitro studies have indicated that occludin, E-cadherin and ZO-1 are critical regulators of intestinal barrier function, and that defects in the junction proteins may facilitate macromolecular flux across the intestinal epithelial barrier $(29,33)$. Although it is unknown whether defective intestinal barrier function is a cause or effect of intestinal inflammation, it is considered to serve a key role in the pathogenesis and relapse of IBD, particularly in CD and UC (34). Therefore, restoring intestinal barrier function may be a potential therapeutic strategy in the treatment of IBD.

Berberine is an established therapeutic for the treatment of gastroenteritis and infectious diarrhea. It is also effective in alleviating experimental colitis in rodents, possibly through anti-inflammatory and anti-oxidative stress activities $(35,36)$. In a human epithelial colorectal adenocarcinoma cell line
(Caco-2) and retinal pigment epithelial cells exposed to TNF- $\alpha$ or IFN- $\gamma$ and TNF- $\alpha$ in vitro, it has been demonstrated that berberine reverses increases in paracellular permeability and preserves the morphological distribution of tight junction proteins, including ZO-1, occludin and claudin-1 (12-14). Furthermore, in a lipopolysaccharide (LPS)-stimulated Caco-2/ macrophage cell co-culture system, berberine-loaded nanoparticles have been demonstrated to inhibit redistribution of ZO-1 and reduce intestinal permeability to FITC-dextran (37). In the present study, berberine inhibited the downregulation of tight junction protein expression in DSS-colitis mice, thus preventing increases in the serum levels of FITC-dextran. These results demonstrate that berberine may restore intestinal barrier function in DSS-colitis mice by preventing the downregulation of tight junction proteins. However, its underlying mechanism of action remains unknown and warrants further study.

Anti-inflammatory and anti-oxidative stress activities of berberine have been demonstrated in experimental colitis in vitro and in vivo $(9,11,38,39)$. Consistent with previous studies, present results demonstrated that berberine treatment in DSS-colitis mice decreased the levels of proinflammatory cytokine mRNA (IL-6, IL-1 $\beta$ and TNF- $\alpha$ ) in colonic tissue, while also decreasing activity of MPO and increasing CAT and SOD activity in colonic tissue and serum. MPO activity is considered to be an indicator of oxidative damage, while SOD and CAT possess anti-oxidative functions (25). It was also demonstrated that berberine inhibited the phosphorylation of STAT3 in colonic tissue of colitis mice. STAT3 is a transcriptional activator and is itself activated by a number of cytokines and growth factors, as well as by oxidative stress $(40,41)$. In T-helper (Th)1/Th17 cells of 2,4,6-trinitrobenzenesulfonic acid-induced colitis mice, it has been documented that berberine reduced the expression of pSTAT3 (36). Therefore, the present results suggest that the regulatory effects of berberine on proinflammatory cytokine and antioxidant enzyme expression may be associated with a decrease in p-STAT3 in DSS-colitis mice. However, the underlying mechanism regarding berberine inhibition of STAT3 phosphorylation warrants further study.

In past studies, DSS induction of colitis in mice disrupted intestinal epithelial barrier function, activated non-lymphoid cells, including macrophages, and stimulated the release of proinflammatory factors, including TNF- $\alpha$, IL-6, and IL-1 $\beta$, resulting in intestinal tissue damage $(42,43)$. Furthermore, it has been observed that macrophages infiltrating the colonic mucosa are activated by proinflammatory factors, which in turn stimulates reactive oxygen species production, in particular superoxide, leading to oxidative stress. Reactive oxygen and nitrogen species produced by macrophages and neutrophils may further aggravate the inflammatory response, causing intestinal mucosal damage in IBD (25). Collectively, these results suggest that macrophages infiltrating the colonic mucosa may be key regulators of intestinal barrier dysfunction, chronic inflammation and oxidative stress in colitis.

Previous studies have demonstrated that there is a significant increase in the number of macrophages within the inflamed intestinal tissue and peripheral blood of patients with CD or UC $(44,45)$. In addition, macrophage depletion in a DSS-induced colitis model was demonstrated to inhibit 
experimental colitis (46). CD68 is a cell surface glycoprotein expressed in a range of mature macrophage populations, including those in the intestinal lamina propria, spleen and peritoneum. Consistent with previous results, the current study demonstrated that CD68 expression, as an indicator of macrophage infiltration, increased in the colonic mucosa of DSS-colitis mice. It has been previously demonstrated that berberine has marked effects on macrophage populations and adjusts the ratio of M2 (anti-inflammatory)/M1 (proinflammatory) macrophage phenotypes among spleenocytes in TNBS-induced mice (36). In DSS-treated mice, berberine inhibited the production of proinflammatory factors in colonic macrophages and epithelial cells, and promoted macrophage apoptosis (11). In addition, in LPS-induced peritoneal macrophage in vitro, berberine inhibited the phosphorylation and nuclear translocation of nuclear factor- $\kappa \mathrm{B}(9)$. In the present study, berberine inhibited macrophage infiltration into the colonic mucosa in DSS-colitis mice. Therefore, berberine may alleviate colitis through the inhibition of macrophage infiltration into the intestinal mucosa.

In conclusion, the present study demonstrated that berberine may alleviate colitis by promoting intestinal barrier function, anti-inflammatory factor production and anti-oxidative stress responses, possibly via the STAT3 signaling pathway. It was also indicated that the key effects of berberine are likely due to its inhibition of macrophage infiltration into the colonic mucosa. However, the underlying mechanisms regarding the regulatory effects of berberine on macrophage infiltration and the primary factors involved in the occurrence and progression of IBD in vivo warrant further study.

\section{Acknowledgements}

The present study was supported by the National Natural Science Foundation of China (grant nos. 81273504, 81473258 and 81402941), the Shanghai Municipal Commission of Health and Family Planning (grant nos. 20144Y0204 and 201540294) and the Science and Technology Commission of Shanghai Municipality (grant no. 15140904600).

\section{References}

1. Deuring JJ, de Haar C, kuipers EJ, Peppelenbosch MP and van der Woude CJ: The cell biology of the intestinal epithelium and its relation to inflammatory bowel disease. Int J Biochem Cell Biol 45: 798-806, 2013.

2. Burisch J and Munkholm P: The epidemiology of inflammatory bowel disease. Scand J Gastroenterol 50: 942-951, 2015.

3. Burisch J, Jess T, Martinato M and Lakatos PL; ECCO-EpiCom: The burden of inflammatory bowel disease in Europe. J Crohns Colitis 7: 322-337, 2013.

4. Sobrado CW, Leal RF and Sobrado LF: Therapies for Crohn's disease: A clinical update. Arq Gastroenterol 53: 206-211, 2016.

5. Iskandar HN, Dhere T and Farraye FA: Ulcerative Colitis: Update on medical management. Curr Gastroenterol Rep 17: 44, 2015.

6. Kong W, Wei J, Abidi P, Lin M, Inaba S, Li C, Wang Y, Wang Z, Si S, Pan H, et al: Berberine is a novel cholesterol-lowering drug working through a unique mechanism distinct from statins. Nat Med 10: 1344-1351, 2004.

7. Jin Y, Khadka DB and Cho WJ: Pharmacological effects of berberine and its derivatives: A patent update. Expert Opin Ther Pat 26: 229-243, 2016.

8. Kumar A, Ekavali Chopra K, Mukherjee M, Pottabathini R and Dhull DK: Current knowledge and pharmacological profile of berberine: An update. Eur J Pharmacol 761: 288-297, 2015.
9. Lee IA, Hyun YJ and Kim DH: Berberine ameliorates TNBS-induced colitis by inhibiting lipid peroxidation, enterobacterial growth and NF-кB activation. Eur J Pharmacol 648: 162-170, 2010.

10. Zhou $\mathrm{H}$ and Mineshita S: The effect of berberine chloride on experimental colitis in rats in vivo and in vitro. J Pharmacol Exp Ther 294: 822-829, 2000.

11. Yan F, Wang L, Shi Y, Cao H, Liu L, Washington MK, Chaturvedi R, Israel DA, Cao H, Wang B, et al: Berberine promotes recovery of colitis and inhibits inflammatory responses in colonic macrophages and epithelial cells in DSS-treated mice. Am J Physiol Gastrointest Liver Physiol 302: G504-G514, 2012.

12. Cui HS, Hayasaka S, Zhang XY, Hayasaka Y, Chi ZL and Zheng LS: Effect of berberine on barrier function in a human retinal pigment epithelial cell line. Jpn J Ophthalmol 51: 64-67, 2007.

13. Zhang DM, Liu HY, Xie L and Liu XD: Effect of baicalin and berberine on transport of nimodipine on primary-cultured, rat brain microvascular endothelial cells. Acta Pharmacol Sin 28: 573-578, 2007.

14. Li N, Gu L, Qu L, Gong J, Li Q, Zhu W and Li J: Berberine attenuates pro-inflammatory cytokine-induced tight junction disruption in an in vitro model of intestinal epithelial cells. Eur J Pharm Sci 40: 1-8, 2010

15. Cao M, Wang P, Sun C, He W and Wang F: Amelioration of IFN- $\gamma$ and TNF- $\alpha$-induced intestinal epithelial barrier dysfunction by berberine via suppression of MLCK-MLC phosphorylation signaling pathway. PLoS One 8: e61944, 2013.

16. Wirtz S, Neufert C, Weigmann B and Neurath MF: Chemically induced mouse models of intestinal inflammation. Nat Protoc 2: 541-546, 2007.

17. Nauseef WM: Myeloperoxidase in human neutrophil host defence. Cell Microbiol 16: 1146-1155, 2014.

18. Moussaoui N, Braniste V, Ait-Belgnaoui A, Gabanou M, Sekkal S, Olier M, Théodorou V, Martin PG and Houdeau E: Changes in intestinal glucocorticoid sensitivity in early life shape the risk of epithelial barrier defect in maternal-deprived rats. PLoS One 9: e88382, 2014.

19. Zhang LC, Li ZZ, Yu YS, Wang ZB, Wei X, Su DF and Li L: Angiotensin-converting enzyme and angiotensin-converting enzyme 2 are involved in sinoaortic denervation-induced cardiovascular hypertrophy in rats. Biol Pharm Bull 34: 1398-1403, 2011.

20. Livak KJ and Schmittgen TD: Analysis of relative gene expression data using real-time quantitative PCR and the 2(-Delta Delta C (T)) Method. Methods 25: 402-408, 2001.

21. Liu WY, Wang ZB, Wang Y, Tong LC, Li Y, Wei X, Luan P and Li L: Increasing the permeability of the blood-brain barrier in three different models in vivo. CNS Neurosci Ther 21: 568-574, 2015.

22. Liang GH and Weber CR: Molecular aspects of tight junction barrier function. Curr Opin Pharmacol 19: 84-89, 2014.

23. Nguyen PM, Putoczki TL and Ernst M: STAT3-activating cytokines: A therapeutic opportunity for inflammatory bowel disease? J Interferon Cytokine Res 35: 340-350, 2015.

24. Xu AT, Li Y, Zhao D, Shen J, Xu XT, Qiao YQ, Zhu MM, Wang TR, Cui Y, Ai LY and Ran ZH: High suppressor of cytokine signaling-3 expression impairs STAT3-dependent protective effects of interleukin-22 in ulcerative colitis in remission. Inflamm Bowel Dis 21: 241-250, 2015.

25. Piechota-Polanczyk A and Fichna J: Review article: The role of oxidative stress in pathogenesis and treatment of inflammatory bowel diseases. Naunyn Schmiedebergs Arch Pharmacol 387: 605-620, 2014.

26. Salvo Romero E, Alonso Cotoner C, Pardo Camacho C, Casado Bedmar M and Vicario M: The intestinal barrier function and its involvement in digestive disease. Rev Esp Enferm Dig 107: 686-696, 2015.

27. Sánchez de Medina F, Romero-Calvo I, Mascaraque $C$ and Martinez-Augustin O: Intestinal inflammation and mucosal barrier function. Inflamm Bowel Dis 20: 2394-2404, 2014.

28. Lee SH: Intestinal permeability regulation by tight junction: Implication on inflammatory bowel diseases. Intest Res 13: 11-18, 2015.

29. Liu WY, Wang ZB, Zhang LC, Wei X and Li L: Tight junction in blood-brain barrier: An overview of structure, regulation and regulator substances. CNS Neurosci Ther 18: 609-615, 2012.

30. Kucharzik T, Walsh SV, Chen J, Parkos CA and Nusrat A: Neutrophil transmigration in inflammatory bowel disease is associated with differential expression of epithelial intercellular junction proteins. Am J Pathol 159: 2001-2009, 2001. 
31. Chen Y, Zhang HS, Fong GH, Xi QL, Wu GH, Bai CG, Ling ZQ, Fan L, Xu YM, Qin YQ, et al: PHD3 stabilizes the tight junction protein occludin and protects intestinal epithelial barrier function. J Biol Chem 290: 20580-20589, 2015.

32. Vivinus-Nébot M, Frin-Mathy G, Bzioueche H, Dainese R, Bernard G, Anty R, Filippi J, Saint-Paul MC, Tulic MK, Verhasselt V, et al: Functional bowel symptoms in quiescent inflammatory bowel diseases: Role of epithelial barrier disruption and low-grade inflammation. Gut 63: 744-752, 2014.

33. Fries W, Belvedere A and Vetrano S: Sealing the broken barrier in IBD: Intestinal permeability, epithelial cells and junctions. Curr Drug Targets 14: 1460-1470, 2013.

34. Merga Y, Campbell BJ and Rhodes JM: Mucosal barrier, bacteria and inflammatory bowel disease: Possibilities for therapy. Dig Dis 32: 475-483, 2014.

35. Chen G, Yang Y, Liu M, Teng Z, Ye J, Xu Y, Cai X, Cheng X, Yang J, Hu C, et al: Banxia xiexin decoction protects against dextran sulfate sodium-induced chronic ulcerative colitis in mice. J Ethnopharmacol 166: 149-156, 2015.

36. Li C, Xi Y, Li S, Zhao Q, Cheng W, Wang Z, Zhong J, Niu X and Chen G: Berberine ameliorates TNBS induced colitis by inhibiting inflammatory responses and Th1/Th17 differentiation. Mol Immunol 67: 444-454, 2015.

37. Wu SJ, Don TM, Lin CW and Mi FL: Delivery of berberine using chitosan/fucoidan-taurine conjugate nanoparticles for treatment of defective intestinal epithelial tight junction barrier. Mar Drugs 12: 5677-5697, 2014.

38. Kawano M, Takagi R, Kaneko A and Matsushita S: Berberine is a dopamine D1- and D2-like receptor antagonist and ameliorates experimentally induced colitis by suppressing innate and adaptive immune responses. J Neuroimmunol 289: 43-55, 2015.
39. Minaiyan M, Ghannadi A, Mahzouni P and Jaffari-Shirazi E: Comparative study of berberis vulgaris fruit extract and berberine chloride effects on acetic acid-induced colitis in rats. Iran J Pharm Res 10: 97-104, 2011

40. Aggarwal BB, Kunnumakkara AB, Harikumar KB, Gupta SR, Tharakan ST, Koca C, Dey S and Sung B: Signal transducer and activator of transcription-3, inflammation, and cancer: How intimate is the relationship? Ann N Y Acad Sci 1171: 59-76, 2009.

41. Bourgeais J, Gouilleux-Gruart V and Gouilleux F: Oxidative metabolism in cancer: A STAT affair? JAKSTAT 2: e25764, 2013

42. Egger B, Bajaj-Elliott M, MacDonald TT, Inglin R, Eysselein VE and Büchler MW: Characterisation of acute murine dextran sodium sulphate colitis: Cytokine profile and dose dependency. Digestion 62: 240-248, 2000.

43. Kitajima S, Takuma S and Morimoto M: Changes in colonic mucosal permeability in mouse colitis induced with dextran sulfate sodium. Exp Anim 48: 137-143, 1999.

44. Te Velde AA, van Kooyk Y, Braat H, Hommes DW, Dellemijn TA, Slors JF, van Deventer SJ and Vyth-Dreese FA: Increased expression of DC-SIGN ${ }^{+}$IL- $12^{+}$IL-18 ${ }^{+}$and CD83 IL-12-IL-18- dendritic cell populations in the colonic mucosa of patients with Crohn's disease. Eur J Immunol 33: 143-151, 2003.

45. Vuckovic S, Florin TH, Khalil D, Zhang MF, Patel K, Hamilton I and Hart DN: CD40 and CD86 upregulation with divergent CMRF44 expression on blood dendritic cells in inflammatory bowel diseases. Am J Gastroenterol 96: 2946-2956, 2001.

46. Ghia JE, Galeazzi F, Ford DC, Hogaboam CM, Vallance BA and Collins S: Role of M-CSF-dependent macrophages in colitis is driven by the nature of the inflammatory stimulus. Am J Physiol Gastrointest Liver Physiol 294: G770-G777, 2008. 